\title{
Changes in the Use of Technology for Language Instruction at the University of Chicago, 2000-2007
}

\author{
Kyung-Hwa Kay Yang \\ McGill University
}

\begin{abstract}
This report is based on my own experience, which I gained through assisting foreign language instruction and linguistics research at the University of Chicago. Over the past few years, technology has become increasingly integrated in language instruction and changed not only the content but also the teaching paradigm. Instructors approach technology quite differently from the past and students are more actively involved in their learning process. Although it is open to dispute whether my observations correlate to general changes in the field of instructional technology, this script, however, may provide a vivid description of a recent situation in this field and suggest a future direction of the working ground.

For over eight years my career has been dedicated to the uses of technology in higher education. I began my career as an intern in the Advanced Multimedia Production Studio at Northwestern University, Illinois in 1999, and since 2000 have served as a manager and multimedia specialist in the Center for the Study of Languages at the University of Chicago ( $U$ of $C$ ). Over my period at the $U$ of $C$, my daily tasks have changed although the crucial role of my position has not. I did and still do define the basis of my job as providing instructors with technology assistance for more effective teaching. Meanwhile, a subtle change has occurred: recently instructors began doing certain tasks which earlier were done by me or other trained staff. One of the reasons for this change is that many instructors now seem to consider technology not only a pedagogical medium but also a tool that they need to learn how to use and incorporate into class. This use of technology often runs parallel with the changing roles of their counterpart, the students.

This report is based on my own experience, which I gained through assisting foreign language instruction and linguistics research at the $\mathrm{U}$ of $\mathrm{C}$. Therefore, it is open to dispute whether my observations correlate to general changes in the field of instructional technology over the past few years. This report, however, may provide a vivid description of a recent situation in this field and suggest a future direction of the working ground.
\end{abstract}




\section{Instructors' Role in Using Technology}

In 2000, my office was full of videotapes, VCRs, and VHS/U-matic edit systems. Instructors brought tapes to make a request for copying or editing. I also found in my desk drawer a Graphic Request Form, which it seems my predecessor had used, along with a CD cover design. She had worked for about two years before I was hired, and was the first multimedia specialist who doubled as a manager. An instructor tells me that technology integration into the curriculum was not a high priority when I was hired, so faculty interaction with my predecessors was usually limited to requesting a task, which this particular instructor could have done herself if she had had more time and resources. Some years ago she started to learn linear and nonlinear video editing. Once she gained confidence and was equipped with a better computer, she preferred working from her own office. This has proven to be a typical case. As a consequence the question for me became how to allocate a limited resource, i.e. the video equipment, to satisfy multiple demands, because the equipment necessary for video editing is not yet as ubiquitous as personal computers and still has to be shared. It does not matter whether this instructor edits video in the most efficient way. She uses the technology in her own way to create pedagogical content and is also open to trying related technology. This instructor's video project influenced many other language instructors who wanted to give students comprehensive learning materials and since then more instructors have contacted me about learning how to use a video camera and a microphone and how to edit videos.

In the age of the Internet, superabundant authentic language materials can be mined on the World Wide Web. It was only a couple of years ago that an instructor knocked at my door, seeking help extracting a segment from an online streaming news file. We worked together to complete the task. A month ago, two instructors came to my office for the same reason. But this time they did not have a specific file they wanted to use. Instead, they brought a pen and paper and pointed out a web site they wanted to use. When I showed them how to do it with a sample file found on the site, one of them said, It's easy! Now two computers are set up so that they can capture and edit streamed video at any time as long as the computers are available. In both of the above cases, students view parts of streaming files selected by their instructors, but the segments are delivered as an integral part of the curriculum in the recent episode while only as ad hoc material in the earlier one. There is another difference which is bigger, I think: in the latter case, the instructors perceived the process of extracting streaming files as simple, and thus manageable. Therefore, from the beginning, they believed that they would not need to spend much time customizing the files. I do not think that they are particularly more tech-savvy than other instructors whom I helped and to whom gave the same instruction before.

Had something changed in the field of technology that would account for the difference between these two instructors and the earlier others? Yes indeed. Nowadays more streaming files are available on the Web and more instructors discover educational value in them. Many educators, especially language instructors, regard the Internet as a useful tool for teaching. (It is only less than twelve years ago that using the Internet made my English teacher uncomfortable because she thought that the information available on the Web was very unreliable, thus she discouraged us from consulting Web sources while doing homework.) But, do the changes in the technology climate alone explain the difference in perception when dealing with new technology? I do not think so, because, if so, many instructors would respond the same way now which seems doubtful. Rather, I believe it is mainly because the two instructors had already established a framework of systematically plugging online streaming files into 
their core curriculum just as they might insert a paragraph from classic texts into their textbook. In this workflow, the only part missing from their standpoint was learning how to cut and paste the streaming files. They wished to place the technology fully under their control.

\section{Paradigm Transition}

Soon after I started working at the U of C, my supervisor, the late Karen Landahl, a professor of linguistics, lamented the poor audio quality of much online content. Most of the online sound files were of poorer quality than regular telephone transmissions. The network bandwidth at many institutions was not sufficient to transmit an acceptable quality of audio and the compression technology was not sophisticated enough to render large audio files without compromising the quality. Furthermore, Dr. Landahl thought that it would be very difficult for students to learn a foreign language with all the mumbling sounds resulting from high compression. Thus, there was reluctance in deploying audio over the Web at that time, although she recognized the potential and value of the Web as an information sharing resource. This decision stemmed from the evaluation that the trendy technology was not yet mature enough to replace the time-tested methods. Most audio materials were then recorded on DAT (Digital Audio Tape) in a soundproof recording studio under professional supervision to bring out the best quality, and students listened to regular cassette tapes copied from the state-of-the-art audio materials. Later in some classes, they were given options to choose online audio materials classified by file size so that students with inferior network environments might be able to access the same content. We, the educators and technologists, serving as content provider in these cases, were challenged to adjust to the rapidly changing conditions.

Ironically however, the quality of audio was not often guaranteed because of scheduling conflicts and insufficient professional assistance, not to mention the long processing time before finally reaching the students' ears. So, instructors began to demand an easier and quicker audio recording process. Consequently, around 2003 an unused CD field recorder was taken out of a closet and into a classroom near instructors' offices or even inside their offices. Technically, DAT might have recorded slightly better quality than $\mathrm{CD}$, but the difference was not significant enough to deter instructors from taking advantage of this newly tested CD recording technology. Its nonlinear track access and high compatibility with other audio equipment made the postproduction easier and quicker. Instructors started to record virtually at any time and edit on their own computers. Some noises from outside were often heard while recording, but it was typically not disruptive enough to make them go back to the studio, either. Additionally, the online course management system became more prevalent at this institution so that making new audio materials available to students was as easy as clicking on a few buttons and uploading them to the server where the system resided. Instructors no longer sought the best quality above all. Instead, they opted for frequent and manageable technology integration despite the possibility of dropping the audio quality to some degree.

The paradigm shift favored producing audio materials with ease over producing audio materials with very high quality that required extensive time and human resources. This shift was cemented by the introduction of a voice-recording tool plugged into the existing online course management system. This tool allows both instructors and students to record audio directly on the system. The quality is only acceptable and editing is not possible at all, but its functional simplicity gained sudden popularity among instructors. As instructors come to play an active role in using technology, it seems to this author that monotonous technology, which has one simple functional interface and can be applicable for various 
activities, will win instructors' heart. High-level technical quality in media is no longer considered a main issue in the selection of technology for courseware development. Rather, technical accessibility and content distribution are given priority. This change may reveal one essential element for successful instructional technology deployment.

\section{The Role of Students in Class}

Even before I started to work at the $U$ of $C$, the first year Chinese class in winter quarter used to begin by viewing the best movie made by students in the previous year. Students were divided into several groups and made two movies, one for winter and one for spring quarter. Part of the curriculum consisted of preparation for creating the movies. While students were writing scripts and performing rehearsals, they learned vocabulary, grammar, and pronunciation. A new learning channel was piped out. Once satisfied with the pre-production, they rolled a camera and cut the video. To complete this assignment they even spent extra hours learning video production. It was an incredible amount of work, especially for beginning students. The language laboratory, which no longer exists now, extended its office hours in the final week to help the students with editing video. In the end, the final class was something like its own Academy Award event. The best movie nominees were honored. After some modification of the curriculum, we see the Academy Award only once per year now. But this vibrant and loved tradition is still passed on to the next generation of Chinese language students, and it is presented to other language instructors as a model of video production activity in class.

A similar approach was implemented for advanced Spanish students about three years ago. A pair of students conducted an interview with native speakers about Hispanic culture and videotaped the interview. After showing the video, the students led the class in an open discussion about the subject matter addressed in the interview. Who is the knowledge provider in this class? Who prepared the teaching materials? Students learned new vocabulary and new cultural content relevant to the video project and combined this new information with what they learned in the class itself. The instructor occasionally interrupted to help students organize their ideas in a logical order or situate their ideas within a larger context. This strategy of open discussion for teaching is not new. Most advanced language courses include student presentations and subsequent discussion without technology involvement. But I think the traditional method hardly sparks such curiosity and the lively discussion that I witnessed in this Spanish class. Listening to Spanish quoted by their classmates is exciting and the video recording technology made it possible. Students' participation, collaboration, and the bi-directional flow of knowledge between instructors and students have become typical of the learning process as technology is fully and actively integrated into the classroom.

Recently I assisted with another class activity that illustrates the above observation. A second year German instructor required her students to produce a fairy tale radio show in small groups as the final assignment. Just like the way the Chinese students worked, the German students were supposed to be scriptwriters, narrators, sound engineers, and producers. When the instructor first came to me to discuss the project, she showed a little anxiety about its outcome because she had never done it before. After each group completed their program, the instructor and I made a master CD with all the final creations. The next day I received a CD of one of the videos that had the group's photo on the CD cover. It was satisfying to witness both the instructor's and the students' enthusiasm for the project. After working with language instructors for many years, I have cultivated my own sense for assessing a technology-enhanced teaching method. I do not understand German, but even I could tell who presented 
the story more engagingly and with clearer pronunciation, thereby making instructor's evaluation easier, and these students apparently read the script loudly and repetitively before recording. Above all, they surely had fun while working on the project.

\section{Summary}

Technology continues to become a mainstream tool in daily communication while instructors continue to exhibit some hesitation to try new tools. The adoption of technology into academic practices tends to occur slowly and conservatively. The process is often characterized as experimental, which requires that instructors take the risk of conducting a new teaching method and endeavor to seek consultation around campus. As a result, I, as technical staff, participate in curriculum design more systematically, not only for content preparation but also for student participation. In this paradigm, three parties play distinctive roles: instructors, as the architects, develop a blueprint, students fill in the content, and technical staff provides tools and technical advice necessary to complete the course. Instructors increasingly want to control technology, and they are no longer lonely trekkers in the jungle of technology. Students readily follow them and, as they do, help to widen the passage so all may successfully reach their final destination. 